\title{
Cavitation during tensile drawing of annealed high density polyethylene
}

\author{
Andrzej Pawlak, Andrzej Galeski \\ Center of Molecular and Macromolecular Studies, Polish Academy of Sciences, \\ Sienkiewicza 112, 90-363 Lodz, Poland
}

Corresponding author: Andrzej Pawlak, tel. +48(42) 6803223

fax: $+48(42) 6847126$

e-mail: apawlak@,bilbo.cbmm.lodz.pl 


\begin{abstract}
The annealing of semicrystalline polymers usually leads to the crystal refinement and thickening. It appears that also the cavitation during tensile drawing is affected. In the uniaxially drawn high density polyethylene a massive cavitation was detected by X-ray scattering in samples previously annealed at $125^{\circ} \mathrm{C}$. The number of voids depends on the annealing time, while their size and orientation depends on the local strain. The cavitation resulted in $30 \%$ increase of volume for annealed samples, strained to 4-5. Cavitation and volume increase were not observed for small and intermediate strains if the polyethylene samples were not annealed. The decrease of drawing rate results in reducing cavitation and also the void stability: at the low strain rate voids were detected during tensile drawing but they disappeared after unloading the sample.
\end{abstract}

Keywords: Cavitation; Plastic deformation; Polyethylene 


\section{INTRODUCTION}

The deformation modes of semicrystalline polymer involve both the amorphous and crystalline components. Initially the deformation is localized in much softer amorphous phase, leading to such effects as interlamellar shear, interlamellar separation or rotation of lamellar stacks. At larger strains the above possibilities exhaust and deformation of crystal begins, mostly by chain slips. The deformation of crystalline phase is irreversible and begins when the yield point of stress-strain curve is observed. Chain slips are possible only in some crystallographic plains and directions for which the critical resolved shear stress is reached. There are other possible mechanisms of crystal deformation such as twinning and - observed in polyethylene - stress induced martensitic transformation, in which the orthorombic structure of undeformed PE is transformed to the monoclinic one. At larger strains, initial unoriented lamelar structure undergoes a transformation to a final, oriented fibrilar structure. The formation of cavities during plastic deformation was observed in many polymers, however, not always and at various ranges of deformation. It was shown that cavitation originates in the amorphous phase and appears at or around the yield point [1-5].

The progress in understanding of voiding mechanism is noticed in the literature published during last 10 years [6-12]. The cavitation in tensile drawing of high density polyethylene was studied by Butler et al. [3,6], Addiego et al [13], Schneider et al. [14] and others. Butler et al. [15] examined in-situ small- and wide-angle X-ray scattering from deformed quenched and annealed PE samples. The cavities were detected at the yield and they were accompanied by low intensity of martensitic transformation. In annealed polyethylene those phenomena were observed exactly at the yield point, however, in the quenched material it occur at advanced stages of cold drawing. However, the martensitic transition must not be associated with cavitation because such transformation was also observed in compression of polyethylene without formation of voids $[8,16]$.

The small angle X-ray scattering (SAXS) patterns indicates that in polyethylene and polypropylene voids are initially elongated perpendicularly to the deformation direction, but at a certain strain the reorientation occurs and in a highly deformed polymer voids are elongated along the deformation direction [9-10,12]. When the lamellar polymer crystals are transformed into fibrilar structure the voids often become large several micrometers in size and may join together.

Usually the cavities are detected by small angle X-ray scattering or by visual observation 
of stress whitening [3,6,17]. G'Sell et al. [13,18] and Lazzeri et al. [19] applied the concept of volume strain to the analysis of plastic deformation of semi-crystalline polymers. It was shown that the measurement of volume may also be a method of detecting the presence of cavities in the material [19]. The determination of relations between mechanical properties and changes in internal morphology by X-ray diffraction is lately easier because the sources of powerful synchrotron radiation are more widely accessible. Results of in-situ SAXS and WAXS measurements were described for many polymers: polypropylene [20,21], poly(vinylidene fluoride) [22], polyamide [23] and polyethylene [24,25]. Usually the studies were focused on the changes in crystalline phase morphology. An example of application of synchrotron radiation to the studies of deformation in polyethylene is work by Hughes et al. [26]. Authors in the rapid tensile test $\left(3 \mathrm{~s}^{-1}\right)$ deformed a "hourglass" profile samples at $35^{\circ} \mathrm{C}$.

The cavitation was observed just after the yield, at the strain of 0.2 . The reorientation of voids was observed in SAXS pattern for large deformations. Similarly to Butler's work [15] the coexistence of orthorombic and small amount of monoclinic phase was noticed for deformed samples, however, the first evidence of monoclinic phase appeared earlier, before the yield point. However, at larger strains only the orthorombic phase existed.

Synchrotron radiation also was used by Schneider et al. [14] for the studies of HDPE deformation. They concluded that a competitive phenomena like crystallite fragmentation and cavitation occur simultaneously at yielding. They proved by the analysis of X-ray scattering intensities that the voids are formed mainly during yielding and at later stages of deformation the number of voids did not increase but existing voids changed their size.

Our previous studies shown that not all polymers cavitates and those that are able to cavitate do this only under some deformation conditions [9-10]. The reason of such behavior is a competition between two mechanisms of deformation of stressed polymer near the yield point: first, the initiation of plastic deformation of crystalline elements - lamella, usually by chain slips, second, the formation of numerous voids in stretched amorphous phase between crystalline lamellae [9]. Therefore, the way in which the deformation proceeds, depends on the relation between the strength of amorphous phase and the resistance of crystals to plastic deformation. In those polymers in which the crystals are small and more defected it is easier to initiate plastic deformation of lamellae than to break the amorphous layer continuity. An example of such polymer is low density polyethylene.

In other polymers, such as high density polyethylene or polypropylene, usually cavitation is first observed, however, its occurence depends in a particular case on morphology and 
deformation conditions. For example we have shown that in the skin of injection molded high density polyethylene sample cavities are formed very easily at the strain below $1 \%$. The cause was the ordered lamellar structure aligned perpendicularly to the surface of the tensile sample. Cavitation was initiated at such low strain because of action of a concentration in the skin layer of the dilatational stress. In the bulk of the sample cavitation was observed at much larger strain, around the yield [10].

The other example of a factor controlling cavitation is the deformation temperature. Polypropylene, usually cavitates at room temperature, however, when the temperature exceeds $40^{\circ} \mathrm{C}$ it deforms without cavitation because the yield stress of crystals decreases with increasing temperature and at the elevated temperatures it becomes lower than the resilience of the amorphous phase [11]. The same polymer may not cavitate at the room temperature if the solidification from melt was rapid and PP crystals are small, defected, showing low yield stress. Such conditions of fast crystallization were obtained by the cooling of molten polypropylene sheets in iced water $[10,12]$.

The above described cavitation process occurs around or at the yield point. It is known that in polymers which do not cavitate around yield point some voiding might be observed at larger strain when the fibrilar structure is formed. However, these voids are not the subject of our paper.

Annealing increases the perfection of crystals and their thickness [8]. Some observations indicate that the sample not cavitating around yield may cavitate after annealing [15]. The main goal of our work described in this paper was the determination of such transition in annealed high density polyethylene and associated changes in cavities shape, size and concentration with increasing deformation. We wanted also to show that the cavitation leads to a significant changes in a polymer volume.

The yield stress of crystals decreases and their ability to plastic deformation by chain slips increases for lower deformation rate. So, it would be expected that for very slow deformation rate a non-cavitational mechanism of plastic deformation will be preferred again. Our earlier investigations for polypropylene have shown that even at the rate as low as $2.5^{*} 10^{-5} \mathrm{~s}^{-1}$ the cavitation was still observed. The limiting drawing rate is not known for polyethylene and other polymers. The results of the determination of the limiting drawing rate are also described in the paper. 


\section{EXPERIMENTAL}

High density polyethylene BASF Lupolen 6021D $\left(\mathrm{M}_{\mathrm{w}} 1.8 * 10^{5}, \mathrm{M}_{\mathrm{w}} / \mathrm{M}_{\mathrm{n}}=7.2\right.$, density 0.960 $\mathrm{g} / \mathrm{cm}^{3}, \mathrm{MFI}=0.26 \mathrm{~g} / 10 \mathrm{~min}$ at $2.16 \mathrm{~kg}, 190^{\circ} \mathrm{C}$ ) was selected for these studies. Samples for experiments were prepared by a compression molding at the temperature of $190^{\circ} \mathrm{C}$, followed by rapid cooling in the water with ice. The $1 \mathrm{~mm}$ thick sheets were produced. Part of the sheets were annealed inside a heating chamber at the temperature of $125{ }^{\circ} \mathrm{C}$. Time of annealing was 1, 3 or 6 hours. The other part was used as the reference material. Dog-bone tensile samples were then cut from these sheets, according to ASTM D 638 Type V standard.

Mechanical tensile properties of HDPE were studied at room temperature using Instron model 5582 mechanical testing machine. Standard engineering drawing rate was $8.3 * 10^{-4} \mathrm{~s}^{-1}$ (i. e. $5 \% / \mathrm{min})$, however, some samples were deformed with lower rates: $6.7 * 10^{-5} \mathrm{~s}^{-1}(0.4$ $\% / \mathrm{min}$ ) and $2.5^{*} 10^{-5} \mathrm{~s}^{-1}$ (i. e. $\left.0.15 \% / \mathrm{min}\right)$. We limited the deformation to engineering strain of $75-100 \%$, much below the strain to break. The actual shape of a sample during deformation was recorded by a "Minolta Dimage" digital camera. A mirror was applied for simultaneous recording of the third dimension of a sample -the thickness. Actual cross-section was calculated from measured dimensions of the sample. For the measurements of the local deformation, black ink marks were placed on the surface of the sample, at the distances of 1 $\mathrm{mm}$. The local strain was determined as the change of distance between marks, $1-1_{0}$, divided by the original distance $1_{0}$. The volume strain was calculated as a change of volume, $\Delta \mathrm{V}=\mathrm{V}-\mathrm{V}_{\mathrm{o}}$, divided by the initial volume, $\mathrm{V}_{\mathrm{o}}$, measured in the most deformed part of a sample.

The small angle X-ray scattering (SAXS) was performed in the synchrotron beamline A2 at Hasylab (Desy) in Hamburg. The goal was the precise detection of cavitation. X-ray wavelength from synchrotron source was $0.15 \mathrm{~nm}$ and the sample to detector distance was 2.6 m. Scattering patterns were collected by a two-dimensional detector operated in $2048 * 2048$ pixel mode. The tensile in-situ observations of voids formation were possible because a custom made tensile testing machine that was fitted to the beamline. The machine performed symmetric drawing of dog-bone shape samples. Signals from a load cell and from the transducer measuring elongation were recorded during the experiment. It was possible to observe the sample and the position of X-ray beam by a TV-camera and to record the consecutive video frames. The deformation was performed with selected rates of $8.3^{*} 10^{-4} \mathrm{~s}^{-1}$, $6.7 * 10^{-5} \mathrm{~s}^{-1}$ and $2.5^{*} 10^{-5} \mathrm{~s}^{-1}$, the same as used in the tensile test, described above. Details of 
experimental synchrotron procedure are described in the paper by Stribeck [21]. The SAXS patterns were also applied for the determination of the long period of undeformed polyethylene samples structure. The position of maximum on curve $I^{*} s^{2}$ vs. s, where I is the intensity of scattering and $\mathrm{s}$ is the scattering vector were used for calculation. The thickness of lamellae was determined using the correlation function method [27,28].

For the determination of sizes of cavities we adopted a method described by Yamashita et al. [29]. The function of $\ln (\mathrm{I})$ vs. $\mathrm{s}^{2}$ was the base for calculation of radius of gyration $\left(\mathrm{R}_{\mathrm{g}}\right)$. Radius of gyration depends on the dimensions of scattering objects. Since the dependence $\ln (\mathrm{I})=\mathrm{f}\left(\mathrm{s}^{2}\right)$ is not linear for deformed HDPE the range of the scattering vector was divided into two parts, in which the dependence $\ln (\mathrm{I})=\mathrm{f}\left(\mathrm{s}^{2}\right)$ is approximately linear. Each of the parts represent another population of cavities, differing in size. The contribution of each group of voids to the total scattering was determined and the radii of gyration was then calculated, beginning with the smallest voids.

The wide angle X-ray scattering (WAXS) camera was used for observations of lamellae orientation. A source of $\mathrm{CuK}_{\alpha}$ radiation, operating at $50 \mathrm{kV}$ and $35 \mathrm{~mA}$ was used. Twodimensional scattering images were recorded by a camera equipped with a Kodak imaging plate. The distance between a sample and recording plate was $5 \mathrm{~cm}$. Exposed imaging plates were analyzed with PhosphorImager SI system (Molecular Dynamics). The stretching of samples was interrupted at selected strain and the sample was fixed in a special frame, which preserved state of strain. The frame with sample was then placed in a holder of WAXS apparatus. Typical time of acquisition was $7 \mathrm{~min}$. For some of HDPE samples WAXS patterns were recorded after relaxation of mechanical stress with the aim of detection of eventual changes of diffraction patterns.

The degree of crystallinity, melting temperature and lamellar thickness were determined from the heat of melting and peak of melting temperature using a DSC 2920 differential scanning calorimeter (TA Instruments). The samples $8-9 \mathrm{mg}$ in weight were heated from $20^{\circ} \mathrm{C}$ to $190^{\circ} \mathrm{C}$ at the rate of $10 \mathrm{~K} / \mathrm{min}$. The crystallinity was calculated from the area under the melting peak, assuming that the heat of fusion for HDPE is $280 \mathrm{~J} / \mathrm{cm}^{3}$ [30]. The length of crystalline stem, $1_{c}$, was determined from equation: $1_{c}=2 \sigma_{e} * T_{m}{ }^{o} /\left(\Delta H_{f} * \Delta T\right)$, where $\sigma_{e}$ is lamellar basal surface free energy (for $\mathrm{PE} \sigma_{\mathrm{e}}=9 * 10^{-6} \mathrm{~J} / \mathrm{cm}^{2}$ [32]), $\mathrm{T}_{\mathrm{m}}{ }^{\mathrm{o}}$ - equilibrium melting temperature (418 K [31]), $\Delta \mathrm{H}_{\mathrm{f}}$-heat of fusion per unit volume (for $\mathrm{PE} \Delta \mathrm{H}_{\mathrm{f}}=280 \mathrm{~J} / \mathrm{cm}^{3}$ ), $\Delta \mathrm{T}=\mathrm{T}_{\mathrm{m}}{ }^{\mathrm{o}}-\mathrm{T}, \mathrm{T}$ melting temperature (position of a maximum on DSC melting peak). The inclination of 
crystalline stems by $35^{\circ}$ to the normal of lamellae surface [32] was taken into account in calculations of lamellae thickness L.

Resistance to degradation of initial and annealed samples was estimated by determination of Oxidation Induction Time, performed according to the EN 728:1997 E standard.

\section{RESULTS AND DISCUSSION}

The compression molding followed by rapid cooling of molten polyethylene in the iced water gave us a material with small, not perfect crystals and with relatively low crystallinity degree. Annealing in controlled thermal conditions was a way to improve the crystals perfection, increase their thickness, and also to increase total crystallinity. The crystalline properties of annealed HDPE are presented in Table I, where DSC and SAXS data are collected. Table I shows that nearly $10 \%$ jump of the crystallinity is seen after $1 \mathrm{~h}$ of annealing. Longer times favor further, but less rapid increase. Maximum degree of crystallinity, $81 \%$, was obtained when the polymer was annealed for $6 \mathrm{hrs}$.

The second important parameter characterizing polymer, mean lamellae thickness, also increases with the time of annealing. Initial, $20 \mathrm{~nm}$ thick crystals thickens to $24 \mathrm{~nm}$ after the one hour of heating at $125^{\circ} \mathrm{C}$. The samples annealed 3 and 6 hours had the same thickness of crystals determined by DSC $-27 \mathrm{~nm}$. SAXS measurements of crystal thickness by applying correlation function method showed a similar tendency to increase the crystal thickness with annealing time, although the obtained values were lower: initial $16 \mathrm{~nm}$ and final $25 \mathrm{~nm}$. The control of thermal stability of polyethylene by Oxydation Induction Time method showed that the resistance of polymer to thermooxydation does not change after annealing and was on an acceptable but not high level of $3 \mathrm{~min}$.

The mechanical properties of unannealed and annealed dog-bone samples were determined during tensile drawing. The results are shown in Fig 1. The engineering stress-strain curves in Fig. 1a represent the drawing of samples annealed for $0,1,3$ and 6 hours and drawn with the same rate of $8.3 * 10^{-4} \mathrm{~s}^{-1}$. Fig $1 \mathrm{~b}$ shows how the change of the strain rate influences mechanical properties of the sample annealed for $3 \mathrm{~h}$. The strain rates of $8.3 * 10^{-4} \mathrm{~s}^{-1}, 6.7 * 10^{-5}$ and $2.5^{*} 10^{-5} \mathrm{~s}^{-1}$ were applied. The annealing process has modified the crystalline structure, as it was shown by DSC (see Table I), and it is not surprise that the mechanical properties of 
HDPE, particularly at yield, depends on the annealing procedure. The mechanical parameters at yield are collected in Table II. The yield stress increases with annealing time from 21.0 MPa for initial specimen to $29.6 \mathrm{MPa}$ for the sample annealed for longest time. The yield strain shows opposite tendency, i. e. it decreases with annealing time from 18.0 to $12.2 \%$. Observed changes in yield behavior are characteristic for the polymers in which the crystallinity increases.

The properties of HDPE at yield also depends on the deformation rate. Decrease of this rate results in lower yield stress and larger yield strain (Fig. 1 b). Yield stress for polyethylene annealed $3 \mathrm{hrs}$ is equal $29.0 \mathrm{MPa}$ when the strain rate is $8.3^{*} 10^{-4} \mathrm{~s}^{-1}$ but only $19.0 \mathrm{MPa}$ when the strain rate is $2.5^{*} 10^{-5} \mathrm{~s}^{-1}$.

Our previous studies [10] have shown that the cavitation in HDPE was not observed for specimens cooled in iced water, for which the yield stress was $19 \mathrm{MPa}$. Limited number of cavities were detected in HDPE samples cooled in water (yield stress of $21 \mathrm{MPa}$ ) and intensive cavitation was seen for polyethylene samples solidified by slow cooling in air. The last samples were characterized by thickest crystals and largest yield stress (26 MPa). These suggested that either the yield stress or crystal thickness may be an indication whether the deformation would involve cavitation or not. The stress threshold between these two possibilities for high density polyethylene seems to be around 21-22 MPa [10]. If the same rules applies here then the cavitation should be expected in all annealed samples tested at standard speed ratio of $8.3^{*} 10^{-4}$, but not in the initial material and not in annealed polyethylene, when the testing rate was very slow (Fig.1b, curve c). Verification of this supposition is presented later.

It is known that the plastic deformation of semicrystalline polymers is often strictly localized and the neck is formed. In our samples the neck was visible when the engineering strain reached of $30-40 \%$. The local strains in neck area were much larger than macroscopic engineering strains. Figure 2 presents relations between the local strain, measured in a most deformed part of neck, and engineering strain.

The linear local strain-engineering strain dependence is seen when engineering strain is below 20\%. For the larger values of engineering strain the localization of strains is visible in all studied samples, however its range depends on the previous annealing of material. The localization of strain is strongest for unannealed sample. When the engineering strain of such sample is $60 \%$, i. e. equivalent to 0.6 in local strain definition, the local strain in most deformed part is 4.5 . The localization of strain decreases with annealing time, however even 
after $6 \mathrm{~h}$ of annealing the neck is formed and local strains are much larger than macroscopic. There are two reasons of strongest deformation in non annealed HDPE. One reason is connected with crystalline phase, the second with amorphous phase. The crystals in non annealed polymer are thinner and more defected, so it is easier to initiate their plastic deformation, leading to higher local deformation. Secondly, non annealed polymer sample consists more amorphous phase, which is also a factor supporting the deformation to larger extension $[33,34]$

Figure $2 \mathrm{~b}$ presents local-engineering strain dependence for the samples annealed $3 \mathrm{~h}$ and deformed with different rates. Linear dependence of engineering and local strains is preserved at the beginning of deformation. At larger applied strain the increase of the local strain was observed, accompanied by the formation of neck. The curves in Fig $2 b$ show also that the decrease of strain rate changes only a little the localization of deformation.

Another aspect of deformation process, observed for annealed samples, is the whitening of a material in a necking zone. Responsible for whitening are submicrometer and micrometer size cavities that are formed during stretching. The whitening of polyethylene was noticed shortly beyond the yield point, i. e. earlier than the necking could be identified. The unannealed specimens do not change their transparency with deformation, i. e. whitening does not occur in this material. Similarly, the samples annealed for $3 \mathrm{hrs}$ were still partially transparent if the strain rate was low, i. e. $2.5^{*} 10^{-5} \mathrm{~s}^{-1}$.

The non uniform strain distribution observed after yielding may be associated with the local changes of volume, that can be measured by video technique for most deformed part of the gauge of a sample. These changes are presented in Figures 3 in the form of volume strain function. There is significant difference in the shape of curve between unannealed and annealed samples. In the unannealed HDPE some small 2-3\% increase of volume is observed for strains of 0.3-0.4. At larger strains the volume decreases and for strains in the range 1-2.5 even some negative values of volume strain are obtained. The negative volume strain was attributed earlier by G'Sell $[35,36]$ to the volume compaction of the amorphous phase due to orientation. Second positive range of the volume strain is observed when strains exceeds 3.5. However, even for strains close to 5.0 the volume of most deformed part of sample is only $15 \%$ larger than the initial one.

The annealed samples showed different behavior of volume strain. The shapes of curves representing annealing times for 1, 3 or $6 \mathrm{hrs}$ are similar, as it is seen in Fig. 3a. The volume 
strain of each of annealed samples quickly increases to 0.04 and with further increase of engineering strain the increase is approximately linear. The $25-30 \%$ increase of volume was recorded for strains of 3-5.

The $30 \%$ increase of volume is much more than it can be explained as a result of Poisson ratio differing from 0.5 . Typical reason of such volume increase is the formation of voids inside the material responsible for the whitening observed in annealed samples.

Figure $3 \mathrm{~b}$ shows the changes of volume for $3 \mathrm{~h}$ annealed samples deformed at selected strain rates. Surprisingly, decreasing the strain rate did not influence significantly the volume strain-strain dependence. Even, for the strain rate of $2.5^{*} 10^{-5} \mathrm{~s}^{-1}$ a significant increase in volume is seen in the deformed sample. The deformation of polyethylene annealed sample at a slower rate is not accompanied by the whitening, however, its volume increases similarly as for higher strain rate. It may suggest that the voids are created in a large amount but their sizes are smaller, significantly below the micrometer range.

Small angle scattering patterns of synchrotron X-ray radiation, measured in situ, during deformation of samples are presented in Figure 4. The images are correlated with engineering stress-engineering strain curves and with local strains, indicated by the numbers below the images. The scattering patterns change significantly with deformation. In the case of the unannealed sample (see Fig 4a) initially only scattering from periodic crystalline structure is visible. It has a shape of ring. The ring preserves its shape to the yield point, however, later at a local strain of 0.25 becomes flattened in the direction parallel to drawing direction and elongated in the direction perpendicular to drawing direction. It means that the long period of structure increases in the direction of drawing and decreases in the perpendicular direction during deformation. The scattering image representing local strain of 0.5 has an additional, more intensive signal, localized near its the center. It is interpreted as a result of limited voiding. The shape and orientation of the scattering from voids change with deformation. The voids are elongated perpendicular to the deformation direction up to a local strain of 0.5 and parallel to the deformation direction for strains of 1.0 and more.

Figure $4 \mathrm{~b}$ illustrates the deformation and SAXS scattering for the HDPE sample annealed for 1 hour. Annealing changes the crystalline structure and the yield strength, however, for local strains below 0.25 the scattering images are very similar to those discussed above. The scattering from cavities is detected first for local strain of 0.25 and the signal becomes much stronger when the local strain is increased to the range from 0.4 to 0.6. Again here the cavities are elongated in direction perpendicular to the deformation direction. Their 
reorientation toward drawing direction begins at a local strain of approx. 0.6. Evidently for each level of deformation the $\mathrm{x}$-ray scattering here is much stronger than the scattering from deformed unannealed sample.

A really strong scattering from voids is observed from samples annealed for $3 \mathrm{~h}$ during deformation (Fig 4c). The scattering from voids elongated perpendicularly to the drawing direction, exceeding significantly the intensity of scattering from crystalline structure, is seen for local strain of 0.12 , i. e. shortly before yielding. The intensity rapidly increases at yielding and then less strongly during further deformation. The scattering streaks from voids change their orientation with further deformation. Initially the voids are oriented perpendicularly to deformation direction. At the local strain close to 0.7 the scattering pattern becomes approximately circular while for larger strain the voids become elongated perpendicularly to the deformation direction. The change of shape of scattering pattern is usually interpreted as reorientation of voids into deformation direction. The second possibility - healing of existing relatively large voids and formation of new oriented in the deformation direction seems to be less probable because of approximately constant total intensity of scattering during reorganization of structure. The scattering from HDPE specimen annealed for $3 \mathrm{hrs}$ is much stronger than the scattering from HDPE sample annealed for $1 \mathrm{~h}$.

The X-ray scattering from sample annealed $6 \mathrm{~h}$ is similar to scattering observed for shorter 3 hrs annealing time, which agrees with the observation that the mechanical properties (yield) and crystal thickness are only a little larger for the samples annealed $6 \mathrm{hrs}$ as compared with $3 \mathrm{hrs}$ annealing. The two differences from the $3 \mathrm{hrs}$ annealed material are that the total scattering intensity is slightly larger and that the reorientation of voids occur later, at a local strain around 0.8-1.0.

The reorientation of voids occurs during the strain softening process, before reaching the plateau on engineering stress-strain curve. The reorientation is detected later for samples with longer annealing time.

The scattering patterns in Fig. 4 are the source of information about voids' sizes, which can be determined from a Guinier type plot by using Yamashita's approach [29]. Vertical scan at a selected strain was the base for analysis of scattering profile and determination of gyration radii. We found that in all of annealed samples the two populations of voids can be separated. In deformed HDPE specimens annealed for $1 \mathrm{~h}$ voids with radius of gyration $\mathrm{R}_{\mathrm{g}}=18$ $\mathrm{nm}$ dominate. There is also $29 \%$ by number of smaller cavities, with $\mathrm{R}_{\mathrm{g}}=6 \mathrm{~nm}$. The samples annealed for 3 and 6 hrs have also two populations of voids characterized by the radii of 
gyration equal 22 and $8 \mathrm{~nm}$, respectively. The measurements for sample annealed $3 \mathrm{hrs}$ (see Fig 4c) showed that the number contents of voids with $\mathrm{R}_{\mathrm{g}}=22 \mathrm{~nm}$ is $89 \%$ at strain of 0.12 , $94 \%$ at strain of 0.14 and $75 \%$ at strain of 0.25 . Very similar results were obtained for the sample annealed $6 \mathrm{~h}$. As example for strain of 0.12 the population of larger cavities with $\mathrm{R}_{\mathrm{g}}=22 \mathrm{~mm}$ was $85 \%$.

The knowledge of radii of gyration is not sufficient for determination of voids sizes if their shape is unknown. In our samples the shape of scattering patterns suggest that the voids are elongated, can be assumed ellipsoidal. The radius of gyration depends on the half axis of ellipsoid, $a$ and $b$, by the formula: $\mathrm{R}_{\mathrm{g}}{ }^{2}=\left(\mathrm{a}^{2}+2 \mathrm{~b}^{2}\right) / 5$. If we assume that the half axis of ellipsoidal void is equal half of amorphous layer thickness, i. e. $b=4 \mathrm{~nm}$ and $\mathrm{R}_{\mathrm{g}}=22 \mathrm{~nm}$ then the second half-axis a is $49 \mathrm{~nm}$, i. e. length of void is $98 \mathrm{~nm}$ while their width equal to the initial amorphous layer thickness. Other observation is that for small strains scattering from cavities in the direction perpendicular to deformation practically does not exist. It means that the size of cavities in the second direction is outside the SAXS detection range, i. e. they are either longer than $60 \mathrm{~nm}$ or shorter than approximately $1 \mathrm{~nm}$. The cavities during reorientation and later enlarge in the direction of drawing, which is visible as a progressive concentration of signal in the center of image, near the beam stop. However, in the perpendicular direction to drawing the scattering appears at the local strain around 0.6 and increases with increasing deformation.

The other objects of our studies by SAXS technique were samples annealed $3 \mathrm{hrs}$ and deformed with decreasing strain rates: $6.7^{*} 10^{-5}, 2.5^{*} 10^{-5} \mathrm{~s}^{-1}$. The measurements with these rates were not possible in situ (as illustrated in Fig. 4), because they were lower than the rate available in the tensile machine connected to synchrotron radiation facilities. The scattering patterns from HDPE sample deformed with slow rates are shown in Fig 5 a,b. These patterns were recorded from differently strained parts of samples after relaxation.

The images for HDPE deformed with the rate of $6.7 * 10^{-5} \mathrm{~s}^{-1}$ look similarly as discussed previously for deformation with the rate of $8.3 * 10^{-4} \mathrm{~s}^{-1}$. Intensive scattering from voids is observed. Further decrease of the strain rate to $2.5^{*} 10^{-5} \mathrm{~s}^{-1}$, i. e. to only $0.15 \%$ of initial gauge length per minute, was effective in decreasing the scattering intensity. The voids were not detected around the yield and also for local strain of 1.1 in so slowly deformed samples after releasing them from clamps (Fig.5b).

The conclusion from Figs. $3 \mathrm{~b}$, and $5 \mathrm{~b}$ is that voids are formed during deformation but they are healed upon unloading the sample. To illustrate clearly the phenomena the following 
experiment was conducted: the sample was drawn with the strain rate of $8.3 * 10^{-4} \mathrm{~s}^{-1}$ and the SAXS pattern recorded during straining at the strain of 0.15 . Then the sample was relaxed by releasing from grips and the SAXS pattern was recorded after 10 min waiting period. Figure $5 \mathrm{c}$ illustrates that although cavities were formed during drawing, no cavities were detected by SAXS after relaxation (Fig 5c,II). It must be mentioned here that such healing of voids is possible only for relatively low strain and low strain rate.

Another interesting observation is made when comparing the parts of SAX scattering originating from crystalline structure. In Fig $5 \mathrm{~b}$ it is visible the orientation of lamellae although the cavities disappeared, while in Fig.5c apparently the lamella orientation and cavities are gone.

The cavities, if present in polymer, are formed inside the amorphous phase between existing crystalline elements. It means that cavitation process and development of cavities shape and size are dependent on the morphological changes of surrounding polymeric matrix. These changes may be studied by analyzing the long period from SAXS experiment and by collecting the data from a wide angle X-ray diffraction.

In SAXS patterns of cavitating polymer the scattering from cavities dominates and overlaps the scattering from periodic crystalline structures - lamellar stacks. For our samples the analysis of long period change with deformation was possible only for non annealed and $1 \mathrm{~h}$ annealed HDPE specimens. The patterns in Fig $4 \mathrm{a}, \mathrm{b}$ were used for this purpose. The results are presented in Fig 6 for three directions of measurements: vertical, horizontal and oblique at $45^{\circ}$. Vertical profile is taken in the deformation direction and represents lamellae stacks perpendicular to deformation direction. Usually, when the polymer has a spherulitic morphology, these crystals are present in equatorial zones of spherulites. Horizontal scattering originates from the lamellar stacks parallel to the deformation direction. The presence of such lamellae is expected in the polar parts of spherulites. The synchrotron measurements gave the following values of long periods for non deformed samples: $25.4 \mathrm{~nm}$ for non annealed PP and $28.2 \mathrm{~nm}$ for 1 hour annealed polymer. The increase in long period is observed on annealing of several polymers and it is plausibly explained by crystal thickening mechanism and sliding diffusion (e. g. [37]).

Figure 6 shows that the values of long period changes significantly with deformation and these changes depend on the direction of measurements. In vertical direction long period increases with deformation for both non annealed and annealed specimens. Opposite tendency is visible in the horizontal direction, where the characteristic lamellae stack distance 
decreases with deformation. These results are expected because the lamellar stacks are strained in equatorial parts and compressed in polar parts of spherulites $[3,6,8,13,15]$. It is interesting to note that the increase of long period in the vertical direction is smaller than the increase of engineering strain. Similar tendency was described recently by Humbert et al. [38] The long period for the polar part lamellae of non annealed samples decreases to $18.5 \mathrm{~nm}$ at 30-35\% strain. The thickness of crystalline layers in non deformed sample, calculated from the crystallinity degree (Table I ) and the initial long period, is $17 \mathrm{~nm}$. It means that the amorphous material between crystals is strongly compressed and also crystals must become thinner due to crystallographic slips. The similar calculations for 1 hours annealed samples, give the values of initial crystal thickness $21.1 \mathrm{~nm}$ and final long period of $25.9 \mathrm{~nm}$. It means that here the thickness of amorphous phase also is significantly decreased during deformation, however, less than for non annealed polypropylene.

In those parts of spherulites which are oriented at $45^{\circ}$ it is assumed that at the beginning of plastic deformation the interlamellar shear occurs, without a change of distance between lamellae. It agrees with the observation that the long period is preserved up to the engineering strain of $20 \%$. For larger strains the distance between crystals in these positions increases, which is probably the result of fragmentation of crystals.

Figure 7 shows WAXS patterns obtained from non annealed and annealed specimens, deformed to selected strains. The measurements were performed after concluding the drawing but the samples remained fixed in a special frame preserving the strain state. The sample annealed for $3 \mathrm{~h}$ was also analyzed after the relaxation. The numbers in Fig. 7 indicate the local strain reached during drawing.

The two circles, which show up in the WAXS patterns for unoriented material and strained to 0.1 (Fig $7 \mathrm{a}, \mathrm{b}$ ), are associated with a diffraction from 110 (inner circle) and 200 (outer circle) lattice planes. In the samples under preserved strain, a third ring, inside the (110) ring appears visible, indexed as (001) of monoclinic form. This form is a result of martensitic transformation of orthorhombic form under stress. The monoclinic form is not visible in the pattern at such strain if the sample was relaxed with free ends (see Fig 7c).

A four-point image of (110) ring is formed after yielding. Also a decrease of intensity of diffraction from (200) plane on meridian and an increase on the equator is observed. The four point (110) pattern is usually formed by (100) chain slip of crystals and is associated with tilting and beginning of lamellae fragmentation $[6,39,40]$. At a larger strain of 0.9 another four point pattern is formed by diffraction on crystals of monoclinic form. 
The monoclinic form is not observed in relaxed samples, even at the strain of 1.3. The effects of plastic deformation represented by changes of diffraction rings are visible in a relaxed polymer for larger local strains than in the same material under stress.

The transition of (110) four point pattern into two arcs is seen for the strain in the range 1.3-2.7. At this strain the concentration of (200) reflections near the equator is also visible. Monoclinic phase with its (001) diffraction ring is detected in deformed and relaxed samples at a strain approaching 2.7 .

Two conclusions from WAXS observations can be drawn: there is no difference in diffraction patterns between non annealed and annealed samples and large part of deformation including martensitic transformation is reversible, even if the sample was strained beyond yield. The WAXS patterns obtained from deformed sample released from grips might not be representative to morphological changes under strain.

\section{CONCLUSIONS}

Annealing of semicrystalline polymers at elevated temperature changes the crystal thickness, crystallinity and perfection of crystals and also the ability of polyethylene to cavitate. We were able by annealing to increase the crystallinity from 66 to $81 \%$ and lamellae thickness, as determined from DSC thermograms, from 20 to $27 \mathrm{~nm}$. It resulted in an increase of the resistance of crystals to plastic deformation. The amorphous phase became thinner, density of entanglements is increased as the same number of entanglements are concentrated in smaller volume and the chains are strained because their fragments were pulled from amorphous phase to be incorporated within crystals. It follows that annealed polyethylene samples have higher yield stress due to thicker crystals while the amorphous phase became more fragile and vulnerable to cavitation.

$\mathrm{X}$-ray scattering experiment confirmed that voids are not generated at yield in non annealed samples, however, even a short annealing promotes cavitation near yielding. Intensity of cavitation depends on the momentary strain and also on the time of annealing. Voids detected by SAXS are in the nanometer range of size but the whitening indicates that also some larger, micrometer size voids are formed during drawing. The shape of cavities evolve with increasing strain: the reorientation of voids is visible during strain softening when the neck is formed. The analysis of SAXS patterns has also shown that in non annealed 
samples, at strains of 0.5 and more, very limited number of voids is formed. These voids appear during final transformation from fragmented lamellae into fibrils. That is also confirmed by the increase in volume (Fig.3a)

The cavitation is a massive phenomena in annealed HDPE specimens, which is best visible as an increase of sample volume. The significant and approximately linear increase of volume is observed beginning with yielding. The volume of sample increases even by $30 \%$ for higher strain. There is no doubt that responsible for the volume increase is cavitation because non annealed and non cavitating polyethylene preserves its volume up to large strains.

Plastic deformation of crystals at a slower deformation rate is easier as that phenomena is rate controlled. With slower deformation rate crystals are deformed easily and cavitation is not initiated. At very slow tensile drawing the cavities may be not stable and heals. In our slowest straining with the rate of $2.5^{*} 10^{-5} \mathrm{~s}^{-1}$ the voids were detected only in stressed samples Martensitic transformation of orthorhombic form into monoclinic form of PE occurs under stress. Martensitic transformation is reversible when the sample is unloaded.

\section{ACKNOWLEDGMENTS}

We thank the Hamburg Synchrotron Radiation Laboratory (HASYLAB) for beam time granted within the frame of the project I 20070061 EC. Also the grant N N508 468834 from the Polish Ministry of Science and Higher Education is acknowledged for the financial support of the work. 


\section{LITERATURE}

1. Duffo P, Monasse B, Haudin JM, G'Sell C, Dahoun A. J. Mater Sci 1995;30(3):701711.

2. Li JX, Cheung WL. Polymer 1999;40(8):2089-2102.

3. Butler MF, Donald AM, Ryan AJ. Polymer 1997;38(22):5521-5538.

4. Castagnet S, Girault S, Gacougnolle JL, Dang P. Polymer 2000;41(21):7523-7530.

5. Nathani H, Dasari A, Misra RDK. Acta Mater 2004;52(11):3217-3227.

6. Butler MF, Donald AM, Ryan AJ. Polymer 1998;39(1):39-52.

7. Gencur SJ., Rimnac CM, Kurtz SM. Biomaterials 2003;24(22):3947-3954.

8. Galeski A. Prog Polym Sci 2003;28(12):1643-1699.

9. Pawlak A, Galeski A. Macromolecules 2005;38(23):9688-9697.

10. Pawlak A. Polymer 2007;48(5):1397-1409.

11. Pawlak A, Galeski A. J Polym Sci Part B Polym Phys (in print).

12. Pawlak A, Galeski A., Macromolecules 2008;41(8):2839-2851.

13. Addiego F, Dahoun A, G'Sell C, Hiver JM. Polymer 2006;47(12):4387-4399.

14. Schneider K, Trabelsi S, Zafeiropoulos NE, Davies R, Riekel C, Stamm M. Macromol Symp 2006;236:241-248.

15. Butler MF, Donald AM, Bras W, Mant GR, Derbyshire GE, Ryan AJ. Macromolecules 1995;28(19):6383-6393.

16. Butler MF, Donald AM, Ryan AJ. Polymer 1998;39(4):781-792.

17. Castagnet S, Girault S, Gacougnolle JL, Dang P. Polymer 2000;41(21):7523-7530.

18. G'Sell C, Hiver JM, Dahoun A. Int J Solids Struct 2002;39(13):3857-3872.

19. Lazzeri A, Thio YS, Cohen RE. J Appl Polym Sci 2004;91(2):925-935.

20. Stribeck N, Nochel U, Funari SS, Schubert T, Timmann A. Macromol Chem Phys 2008;209:1992-2002.

21. Stribeck N, Nochel U, Funari SS, Schubert T. J Pol Sci Part B Polym Phys 2008;46(7):721-726.

22. Wu J, Schultz JM, Yeh F, Hsiao BS, Chu B. Macromolecules 2000;33(5):1765-1777.

23. Murthy NS, Bednarczyk C, Moore RAF, Grubb DT. J Polym Sci. Part B Polym Phys 1996;34(5):821-835.

24. Jiang Z, Tang Y, Men Y, EnderleHF, Lilge D, Roth SV, Gehrke R, Rieger J. Macromolecules 2007;40(20):7263-7269. 
25. Men Y, Rieger J, Homeyer J. Macromolecules 2004;37():9481-9488.

26. Hughes DJ, Mahendrasingam A, Oatway WB, Heeley EL, Martin C, Fuller W. Polymer 1997;38(26):6427-6430.

27. Strobl GR, Schneider M. J Polym Sci Polym Phys Ed 1980;18(6):1343-1359.

28. Goderis B, Reynaers H, Koch MHJ, Mathot VBF. J Polym Sci Part B Polym Phys 1999;37(14):1715-1738.

29. Yamashita T, Nabeshima Y. Polymer 2000;41(16):6067-6079.

30. Hoffman JD. Polymer1982;23(5):656-670.

31. Psarski M, Piorkowska E, Galeski A. Macromolecules 2000; 33(3):916-932.

32. Patel D, Bassett DC. Polymer 2002;43(13):3795-3802.

33. Séguéla R. Macromolecular Materials and Engineering 2007;292:235-244.

34. Humbert S, Lame O, Vigier G. Polymer 2009;50(15):3755-3761.

35. Quatravaux T, Elkoun S, G'Sell C, Cangemi L, Meimon Y. J Polym Sci Part B Polym Phys 2002;40(22):2516-2522.

36. Cangemi L, Elkoun S, G'Sell C, Meimon Y. J Appl Polym Sci 2004;91(3):1784-91.

37. Hikosaka M, Watanabe K, Okada K, Yamazaki S. Adv Polym Sci 2005;191:137-186

38. Humbert S, Lame O, Chenal JM, Rochas C, Vigier G. J Pol Sci Part B Polym Phys, to be published.

39. Hiss R, Hobeika S, Lynn C, Strobl G. Macromolecules 1999;32(13):4390-4403

40. Galeski A, Bartczak Z, Argon AS, Cohen RE. Macromolecules 1992;25(21):57055718 
Table I. Crystalline structure of HDPE samples annealed at $125^{\circ} \mathrm{C}$ as revealed by DSC and SAXS.

\begin{tabular}{|l|l|l|l|l|l|l|}
\hline $\begin{array}{l}\text { Annealing } \\
\text { time }[\mathrm{h}]\end{array}$ & $\begin{array}{l}\text { Melting } \\
\text { temperature } \\
{\left[{ }^{\circ} \mathrm{C}\right]}\end{array}$ & $\begin{array}{l}\text { Enthalpy } \\
\text { of } \\
\text { melting } \\
{[\mathrm{J} / \mathrm{g}]}\end{array}$ & $\begin{array}{l}\text { Crystallinity } \\
{[\%]}\end{array}$ & $\begin{array}{l}\text { Stem } \\
\text { length } \\
{[\mathrm{nm}]}\end{array}$ & $\begin{array}{l}\text { Lamellae } \\
\text { thickness } \\
\text { by DSC } \\
{[\mathrm{nm}]}\end{array}$ & $\begin{array}{l}\text { Lamellae thickness } \\
\text { by SAXS } \\
\text { correlation function } \\
{[\mathrm{nm}]}\end{array}$ \\
\hline 0 & 133 & 191 & 66 & 24.5 & 20 & 16 \\
\hline 1 & 135 & 216 & 75 & 29.4 & 24 & 21 \\
\hline 3 & 136 & 224 & 78 & 33.0 & 27 & 24 \\
\hline 6 & 136 & 233 & 81 & 33.0 & 27 & 25 \\
\hline
\end{tabular}

Table II. Yield properties of HDPE annealed samples.

\begin{tabular}{|l|l|l|l|}
\hline Annealing time $[\mathrm{h}]$ & Strain rate $\left[\mathrm{s}^{-1}\right]$ & Yield stress $[\mathrm{MPa}]$ & Yield strain $[\%]$ \\
\hline 0 & \multirow{2}{*}{$8.3 * 10^{-4}$} & 21.0 & 18.0 \\
\cline { 4 - 4 } 1 & & 26.4 & 16.5 \\
\cline { 4 - 4 } 3 & & 29.0 & 12.7 \\
\cline { 3 - 4 } 6 & $6.7 * 10^{-5}$ & 29.6 & 12.2 \\
\hline 3 & $2.5 * 10^{-5}$ & 23.8 & 16.0 \\
\hline 3 & & 19.0 & 19.3 \\
\hline
\end{tabular}




\section{FIGURE CAPTION}

1. Typical engineering stress-engineering strain dependence for HDPE. (1a) Different annealing time: a) $6 \mathrm{~h}$, b) $3 \mathrm{~h}$, c) $1 \mathrm{~h}, \mathrm{~d}$ ) not annealed; (1b) Different strain rates of samples annealed for $3 \mathrm{hrs}$ at $125^{\circ} \mathrm{C}$ : a) $8.3 * 10^{-4} \mathrm{~s}^{-1}$, b) $6.7 * 10^{-5} \mathrm{~s}^{-1}$, c) $2.5^{*} 10^{-5} \mathrm{~s}^{-1}$.

2. Dependence of strain in most deformed parts of HDPE samples as a function of engineering strain: (a) samples annealed at different time and stretched with the rate of $8.3 * 10^{-4} \mathrm{~s}^{-1}$, (b) samples annealed $3 \mathrm{~h}$ and stretched with different rates.

3. Volume strain as a function of strain: (3a) different annealing time, (3b) different strain rates of samples annealed at $125^{\circ} \mathrm{C}$ for $3 \mathrm{hrs}$.

4. SAXS scattering measured in situ during tensile drawing with rate of $8.3^{*} 10^{-4} \mathrm{~s}^{-1}$ : a) unannealed sample, b) sample annealed for $1 \mathrm{~h}$, c) sample annealed for $3 \mathrm{~h}, \mathrm{~d}$ ) sample annealed for $6 \mathrm{~h}$. Numbers below SAXS patterns represent local strain in sample at the moment of SAXS pattern recording. Arrow shows the deformation direction.

5. Scattering patterns from HDPE samples annealed $3 \mathrm{~h}$ and deformed with rate of $6.7^{*} 10^{-5} \mathrm{~s}^{-1}$ (a), $2.5^{*} 10^{-5} \mathrm{~s}^{-1}(\mathrm{~b})$ and $8.3 * 10^{-4} \mathrm{~s}^{-1}(\mathrm{c})$. The numbers below images represents local strain. The samples were deformed in vertical direction. SAXS patterns of samples in (a) and (b) were recorded after relaxation. SAXS pattern of the sample in (c) was recorded under stress (I) when the strain was 0.15 and after relaxation (II).

6. Change of long period for unannealed (a) and $1 \mathrm{~h}$ annealed HDPE (b) samples during deformation with the strain rate of $8.3^{*} 10^{-4} \mathrm{~s}^{-1}$, determined from in situ recorded SAXS patterns in vertical $(\bullet)$, horizontal $(\circ)$ and 45 degree directions $(\boldsymbol{\nabla})$.

7. WAXS patterns from HDPE samples: a) non annealed sample, deformed and kept at fixed strain, b) sample $3 \mathrm{~h}$ annealed, deformed and kept at fixed strain, c) $3 \mathrm{~h}$ annealed sample, deformed and relaxed with free ends. Numbers indicate local strain. Deformation direction was horizontal. 



Fig. 1 

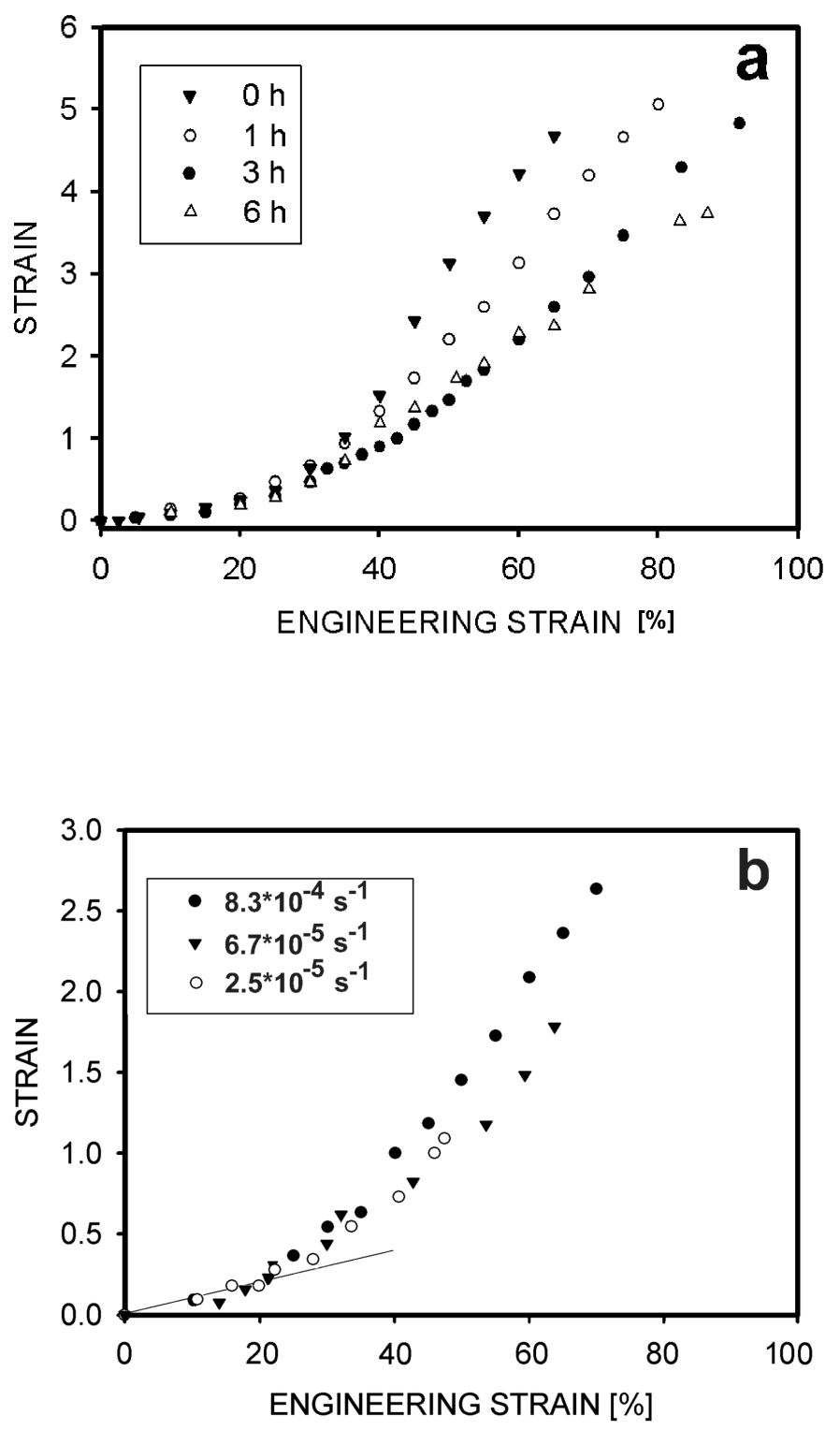

Fig. 2 

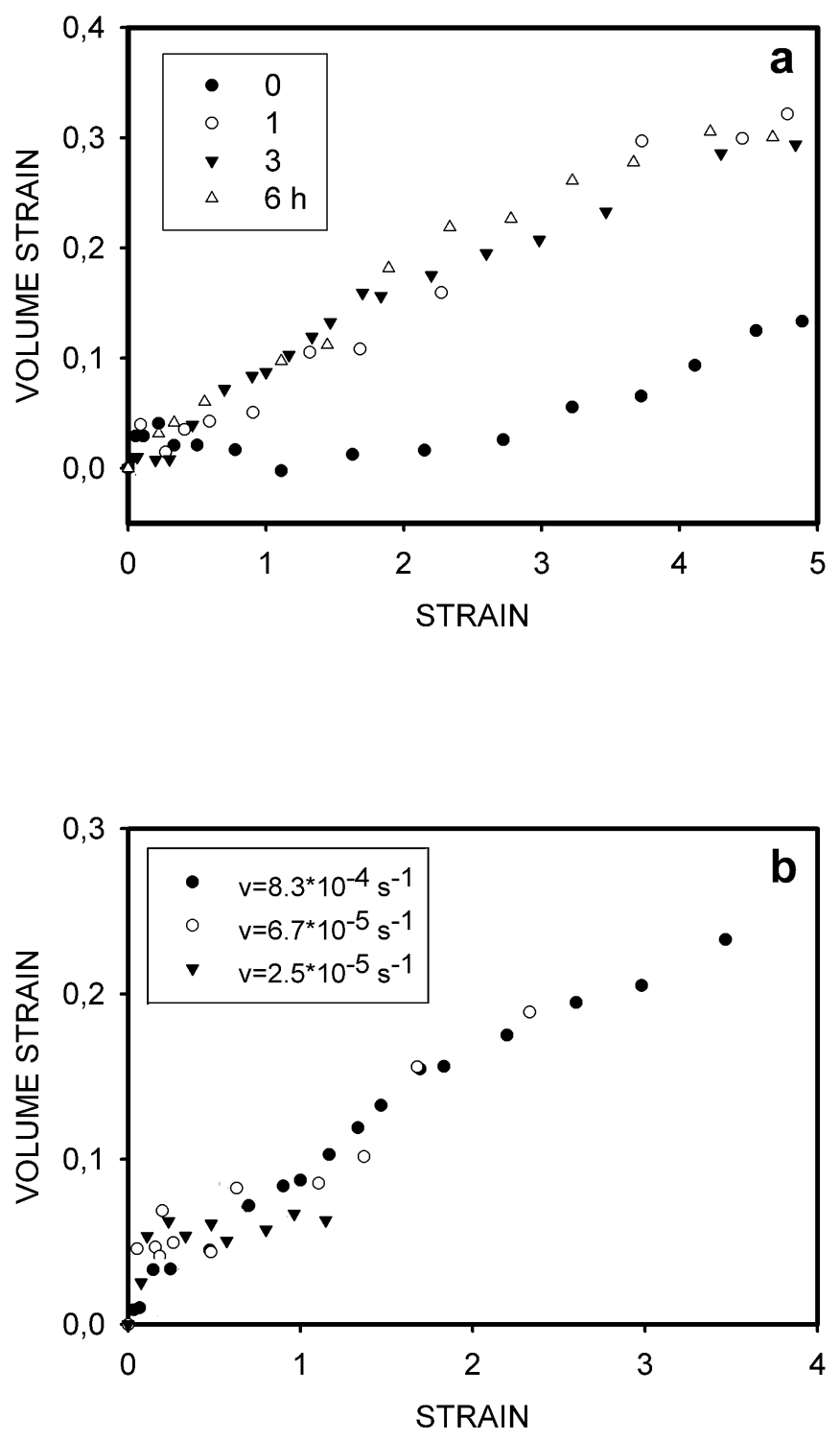

Fig. 3 
a

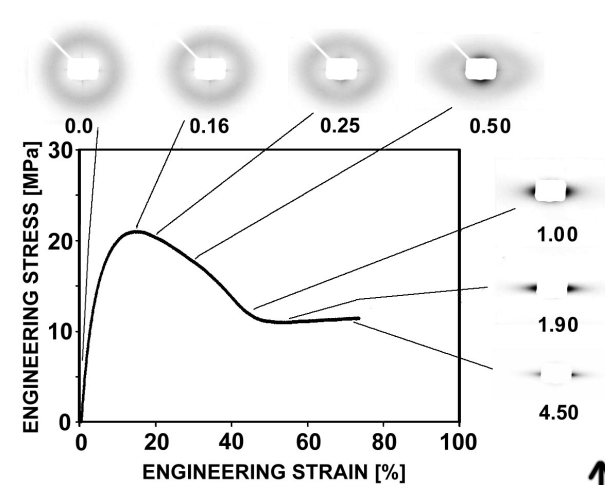

\section{C}

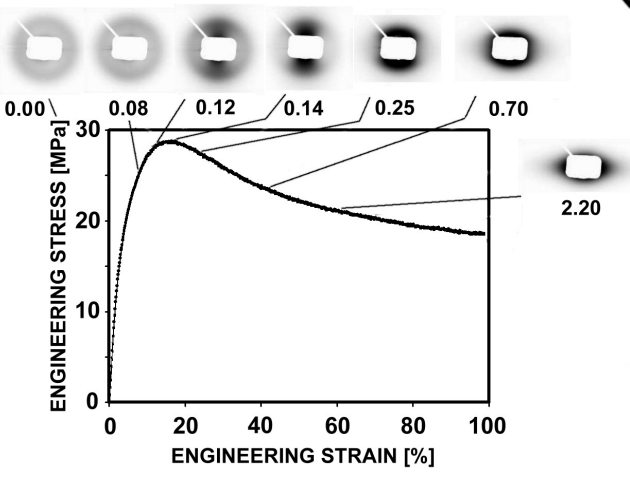

b

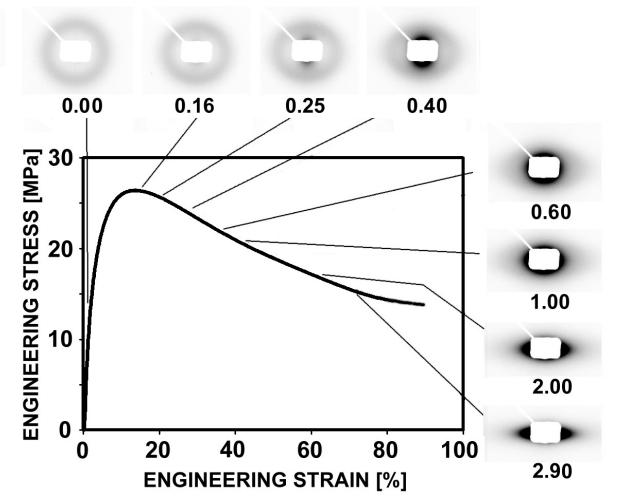

d

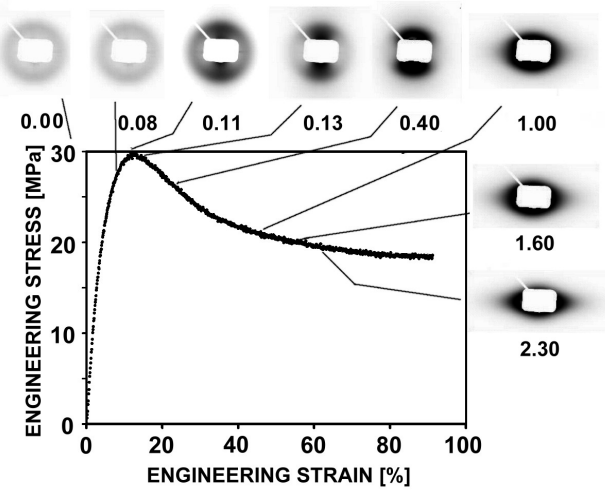

Fig. 4

a)

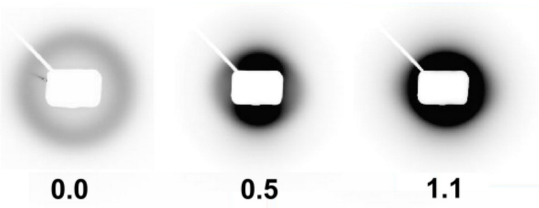

b)

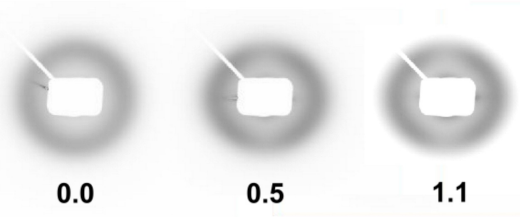

c)

Fig. 5 

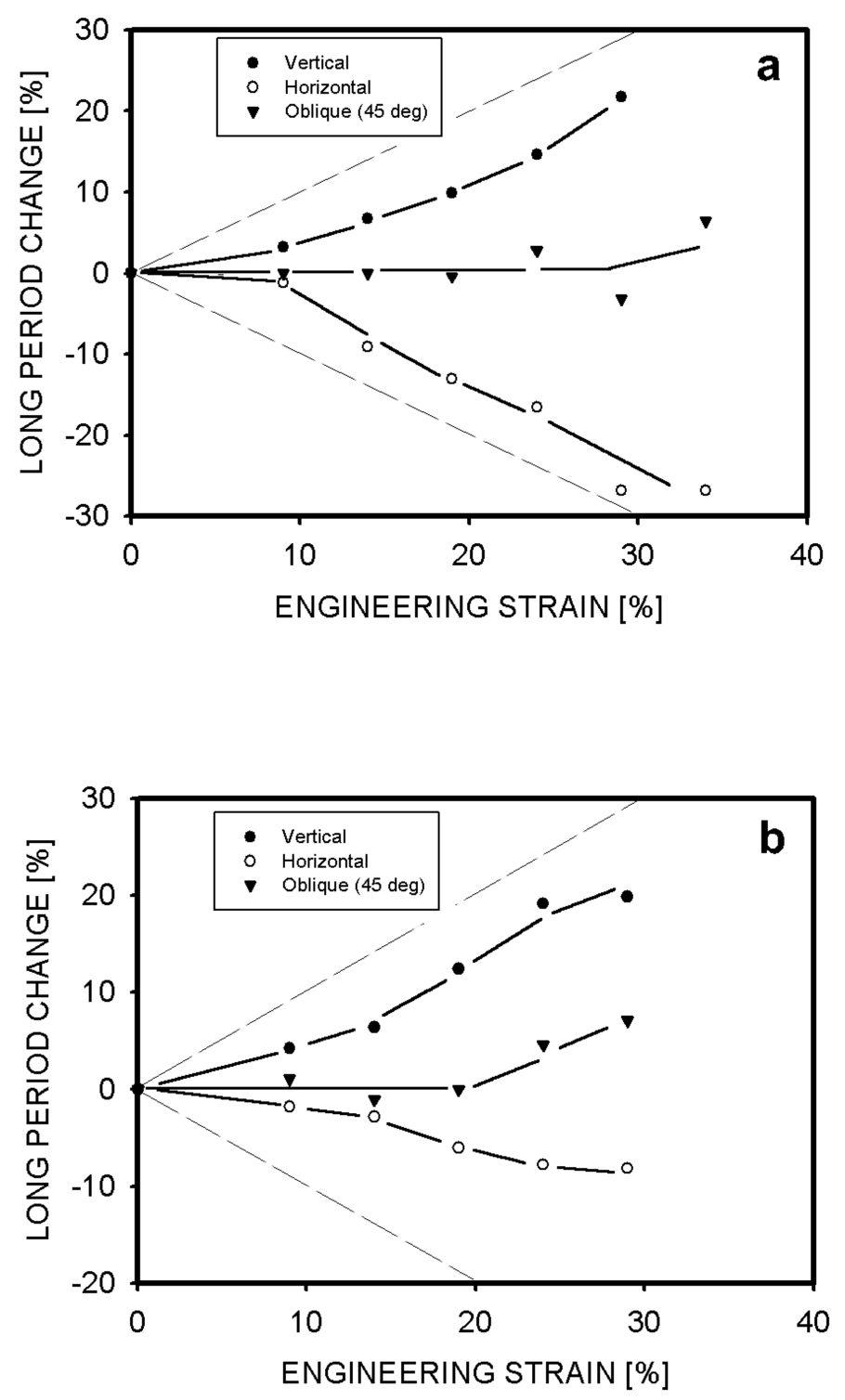

Fig. 6 
a
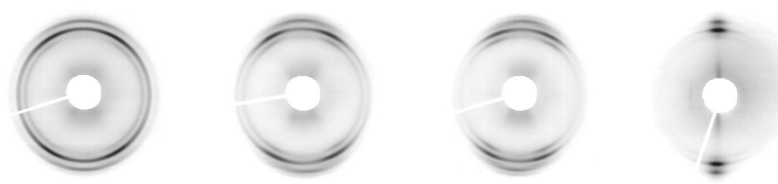

b


c

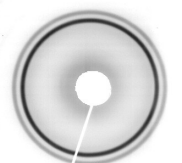

0.1

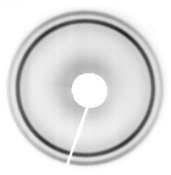

0.5

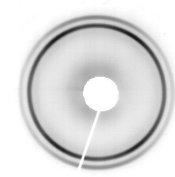

0.9

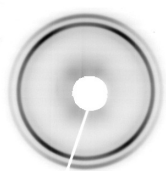

1.3

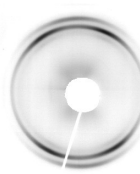

2.7

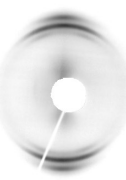

4.5

Fig. 7 
